\title{
Realising potential and recognising paradox: The national induction and mentoring project
}

\author{
Frances Langdon, Peter Lind, \\ Cynthia ShaW ANd ERIN PiLcher
}

\section{Abstract}

Although comprehensive policy and resourcing of beginning teacher induction and mentoring can improve teacher retention and quality, there is growing recognition that combining on-site leadership and policy is integral to providing effective learning for teachers. This has led to an increased interest in melding policy and resources with school and service leadership to promote consistency of beginning teachers' induction and mentoring experiences. This article describes and provides insights into a project involving four pilots which are trialling the draft national guidelines for effective induction programmes and mentor teacher development. An external evaluation across the four pilots has revealed that national guidelines can be a positive lever for effecting change in induction and mentoring practices. Implementing such change nationally will require leaders to take seriously an educative, transformative approach to learning for both beginning teachers and their mentors.

\section{Introduction}

7 he rapid international expansion of policy and resources to support beginning teacher induction and mentoring recognises

1 that such programmes are capable of addressing teacher retention and quality concerns. While some teacher attrition is normal, and in some cases desirable, large attrition rates, particularly of early career teachers, are costly and lead to teacher shortages. A response to this has been the implementation of induction and mentoring programmes to reduce teacher attrition (Smith \& Ingersoll, 2004). In assessing the evidence on effective teacher induction and mentoring, Strong (2009) found that the more comprehensive the support, the 
"less likely teachers are to quit" (p. 102). This is reinforced in the findings of a New Zealand study (Cameron, Dingle, \& Brooking, 2007).

Most induction programmes focus on support for beginning teachers by providing advice, guidance, information and psychological support to enable them to survive and accommodate the stressors of a new and demanding job (Gold, 1996). Yusko and Feiman-Nemser (2008) argue that "this support-orientated approach is a humane response to the challenges of beginning teaching, but does not offer a comprehensive rationale for serious induction" (p. 925). Focusing primarily on support and advice overlooks the fact that beginning teachers are still learning to teach. Even the best preservice teacher education programme cannot fully provide all the learning, knowledge and skills required of a teacher. It is only on the job that the actual intellectual and emotional complexity of teaching becomes a reality, and it is only in context that certain understandings and skills can be developed. Serious, on-site, comprehensive induction has the potential to help beginning teachers develop effective teaching practice when support is blended with educative mentoring, learning, and assessment (Yusko \& Feiman-Nemser, 2008).

There is growing interest in the possibilities inherent in comprehensive induction and mentoring. Britton, Paine, Pimm and Raizen (2003) provide an informed and useful description of the term in their study which examined induction systems located in five countries (France, Japan, New Zealand, China [Shanghai] and Switzerland). They differentiate 'limited induction', which focuses on teacher orientation, support, enculturation and retention, from 'comprehensive induction'. The latter, they describe as supported by policy that requires participation, is more than one year in length, and is goal-orientated with adequate resources that provide substantial paid time to meet these goals. In their view, comprehensive induction is a planned system designed to involve complementary providers and provide good working conditions and training to promote career learning that enhances teaching quality. Likewise, mentoring is played out differently in different contexts. The Teachers Matter study (Organisation for Economic Co-operation and Development, 2005) found that in most countries the mentor's role is to provide support and fix deficits in, for example, classroom management. Norman and Feiman-Nemser (2005) advocate an educative form of mentoring. In 
this approach the mentor has a "vision of good teaching" (p. 608) and regards new teachers as learners who need to think about how to develop principled teaching practice. This contrasts with a narrow view of mentoring which has the primary purpose of "easing the new teachers' entry and helping with the immediate questions and uncertainties that arise when a new teacher enters the classroom for the first time" (Norman \& Feiman-Nemser, 2005, p. 680).

Since 1985, New Zealand has been known as a country committed to providing comprehensive induction for beginning teachers. The induction system has been described internationally as "state of the art" (Wong, 2005, p. 42). Schools and early childhood education services are provided with policy, guidelines, funding, resources, and time. Beginning teachers receive on-site mentoring to facilitate the gathering of evidence that they need to meet the national standards for full teacher registration. Yet despite the comprehensive support, research into induction practices since 1989 has shown variability in the quality of the induction and mentoring experienced by beginning teachers (Aitken, Ferguson, Mcgrath, Piggot-Irvine, \& Ritchie, 2008; Battersby, 1989; Cameron, 2006, 2007, 2009; Cameron et al., 2007; Dewar, Kennedy, Staig, \& Lois, 2003; Mansell, 1996; Renwick, 2001).

To address the variability of beginning teachers' induction experiences, in 2006 the New Zealand Teachers Council (the Council) launched a major research programme, Learning to Teach, to investigate the quality of induction and mentoring provided in New Zealand to provisionally registered teachers (PRTs). This comprised three stages: a literature review (Cameron, 2007), a national survey of PRTs (Cameron et al., 2007), and in-depth case studies (Aitken et al., 2008). Cameron et al. (2007, p. 108) provided evidence that while novices felt personally welcomed and valued, "many did not receive the level of support, mentoring and assessment to which they were entitled". A significant proportion of teachers - 22 percent of early childhood education teachers, 21 percent of secondary and 14 percent of primary teachers considered that they had been left alone to "sink or swim".

It seems, paradoxically, that while the New Zealand system is recognised as comprehensive and capable of providing effective induction, appropriate resourcing and programmes are not reaching all new teachers. This poses a significant challenge as beginning teachers' learning is primarily experienced on the job. They rely on their school or service to promote high quality support and practice. 
The success of induction and the benefits of formal learning experiences and mentoring are "limited if a school's leadership and culture send contrary messages regarding performance standards" (Sparks, 2005). Further, Sparks argues that the mentor-beginning teacher relationship can simply serve "to further entrench current practice and heighten the resistance to serious reform of teaching and learning" unless mentors are carefully selected and trained to act as "embodiments of the desired future of teaching and learning in the school" (pp. 242-243). This cautionary message applies equally to early childhood education services. For induction and mentoring programmes to be effective, they need to be integrated into effective school or service professional development structures and culture, thus embedding the notion that induction is an introduction to careerlong professional learning and development to strengthen teaching and student learning (Cameron, 2007).

In response to the questions raised by the Learning to Teach research about inconsistency of beginning teachers' induction experiences and mentoring capability, the Council developed draft guidelines for induction and mentoring programmes (New Zealand Teachers Council, 2009a). These guidelines aim to shift the focus of induction from a system that Britton et al. (2003, p. 334) found is well crafted to support the "status quo" and teachers "fitting-in", to a transformative, educative approach to mentoring beginning teachers. In a national project, the Induction and Mentoring Pilot Project (IMPP), the Council is trialling the draft guidelines in early childhood, primary/intermediate, secondary and Māori-medium sites.

This article discusses the IMPP and presents the key findings to date from an independent evaluation of the project (Teague \& Sankar, 2010). The insights gained from the first year of the project are discussed, and it is argued that policy and resourcing alone are not enough for effective induction and mentoring; it also requires organisational leadership that promotes effective teaching, teacher learning communities, and educative mentoring. When schools and early childhood education services meld resourcing and effective leadership with policy and guidelines, the likelihood of improving beginning teacher experiences of effective induction and mentoring will increase. Effective induction should in turn lead to improved teaching practice; however, there are challenges that must be addressed. 


\section{The New Zealand Teachers Council Induction and Mentoring Project}

\section{Background}

In New Zealand, existing policy and resourcing for induction of PRTs is focused primarily on ensuring that these teachers have adequate "advice and guidance" with opportunities to be appraised and assessed against the teacher registration standards. The Council was aware of the literature discussing the comprehensive, educative approach to induction (Feiman-Nemser, 2001) that showed what a potentially powerful lever this process could be to strengthen the profession. However, the findings from the Council's Learning to Teach research programme indicated that many PRTs did not have access to a structured induction programme, and that much of the mentoring was little more than the provision of emotional support and enculturation into the workplace - a limited approach, as discussed in the first section of this article. In addition, in New Zealand early childhood education teachers, employed in services for children from birth to five years of age, were becoming qualified and registered as part of a government strategic plan for early childhood education. Early childhood education services, with the exception of kindergartens, did not have the school sector's tradition of supporting newly qualified teachers. These services were looking to the Council for a clear direction on how to develop strong induction and mentoring leadership.

The Learning to Teach research programme provided a sound evidence base for moving policy and practice towards a more consistent and comprehensive approach to induction of PRTs. The literature review (Cameron, 2007) provided a description of current practices and examined models of "best practice". This was followed by a random survey of recent PRTs (Cameron et al., 2007) to probe the nature of the induction and mentoring support they had received. It found that many PRTs were unaware of the criteria used to guide their programme and for assessing and providing feedback on their performance. The survey set up a framework of ideal practices drawn from the literature review, and found that mentors were not, on the whole, providing the kind of critical, evidence-informed feedback which characterises an educative model of mentoring. The survey was followed by the successful case studies which looked at "good" induction programmes in a range of 
settings (Aitken et al., 2008). The studies teased out in the New Zealand setting what was seen to be the conditions needed to provide comprehensive models of induction for PRTs.

The findings from the research programme contributed to the development of the draft guidelines for induction and mentoring designed to shift induction and mentoring practices rather than embed existing approaches. For example, the guidelines include a statement that an educative model of induction and mentoring should be transformative in nature and should benefit the full range of learners in New Zealand schools and early childhood education services. The draft set of skills, knowledge and attributes for mentor teachers focus on abilities such as providing critical, evidence-based feedback to PRTs, rather than enculturation to the particular workplace or "handy tips" based on the preferred practices of the mentor teacher.

\section{Setting up the pilot project}

The next step was to trial the draft guidelines using a range of models to address issues that surfaced in the literature - both to mitigate barriers and enhance good induction and mentoring practices. The literature had emphasised the key role of the mentor teacher in facilitating the "learning on the job" of the beginning teacher and the need to carefully select and train mentor teachers for this important role. Therefore, the Council sought proposals which could provide sustainable models of mentor teacher development. Contracts were awarded to organisations trialling models of induction and mentoring and mentor teacher development in four different sectors: early childhood education services, primary/intermediate schools, secondary schools, and Māorimedium settings. Together, these four pilots comprise the IMPP.

A research component was required within each pilot to track the development of the individual pilot models and to explore their effectiveness. However, because the Council was seeking strong, credible evidence to inform future developments in national policy, it also commissioned an independent evaluation of the pilots to test the viability of the draft guidelines across the pilots and to probe the ability of the pilots to deliver sustainable programmes that addressed the issues emerging from the research programme and other literature.

The Council's primary goal for PRT induction and mentoring programmes is to ensure that the programmes are high quality, responsive to PRTs' needs, supported and promoted by professional 
leaders, and implemented by appropriately trained mentor teachers. These elements collectively contribute to supporting PRTs to become effective teachers and achieve equitable learning outcomes for all learners, and they informed the design and development of the IMPP.

The IMPP is unique in its scale and breadth as it has involved a wide cross-sector of the education community in both the execution and management of the project. This approach is a deliberate strategy because if changes are to happen in New Zealand's policy environment of self-managing schools and independent early childhood education services, a broad consensus and a strong commitment will be needed to make the desirable changes.

The next section describes the individual pilots. At the time of writing, the one-year early childhood education pilot (2009) was completed, the primary/intermediate and secondary school pilots (2009-10) were midway through a two-year term and the Māori medium pilot had been extended from an initial one-year term to 18 months, to be completed in August 2010. The different timeframes of the pilots have provided some challenges in terms of managing and evaluating the programme; however, these reflect the different approaches to the provision of induction and mentoring. It should also be noted that each pilot obtained ethical approval from their institution before commencement. Practices and process, based on the Treaty of Waitangi principles, are evident in each project.

\section{The National Pilot Project}

\section{The Early Childhood Education Pilot}

Ka rongo ka wareware, ka kitea ka mahara, ka hangaia ahua, ka marama ahau

I hear and I forget, I see and I remember, I do and I understand

The early childhood education pilot was based on a clear view of continuing professional learning focused on reflection, collaboration, critical enquiry, openness to challenge and change, and transformational in outcomes for learners. Led by New Zealand Kindergartens Inc., this pilot was an example of practitioners leading and learning alongside practitioners, drawing on external expertise to push the boundaries of professional learning. The collaborative nature 
of the pilot has been evident on many levels, particularly the visibility of mentor teachers learning alongside the PRTs.

Participants. The pilot involved 60 PRTs and 51 mentor teachers from five kindergarten associations clustered into three regions across New Zealand (Wellington/Ruahine, Nelson, and Dunedin/Southland). Mentors from Te Tari Puna Ora o Aotearoa/New Zealand Childcare Association were part of the Wellington/Ruahine regional group, and PRTs from education and care centres participated in Nelson. Kindergarten senior teachers in the pilot regions attended the workshops and supported the focus group process. An advisory group was also formed to guide the establishment of action learning cycles from each phase of the pilot, and its members were active participants in the research process.

The professional development intervention. The main components of this one-year pilot were firstly, that PRTs and mentor teachers from the three regions participated in a series of regional workshops in 2009 , and secondly, an online resource was developed to provide ongoing support. There were 16 workshops in total, including a twoday national hui for mentors midway through the pilot. The workshops focused on unpacking the draft induction and mentoring guidelines, especially the dispositions and skills of mentor teachers, the Registered Teacher Criteria (New Zealand Teachers Council, 2009b), goal setting and documentation. The content of each workshop reflected feedback from the previous workshop, providing skill development in critical areas such as observing teacher practice and giving feedback, evidence-informed reflective practice, making professional judgments, managing difficult conversations, and listening skills. The pilot drew on the experience and expertise of the professional senior teacher teams in kindergarten associations. Between workshops, senior teachers provided follow-up in their regions, drawing on the workshop content to focus on both the PRT and the mentor teacher's practice.

Research. The overarching research question for this pilot was "How does the implementation of the induction and mentoring programme make a difference to the mentors' and PRTs' learning and teaching?" The emerging research findings were used to inform the action learning process of the pilot and each new stage of development. 


\section{The Primary/Intermediate Education Pilot}

Ehara taku toa I te toa takitahi, engari he toa takitini

My strength is not that of a single warrior but that of many

The University of Auckland, which conducted the primary/intermediate education pilot, took a whole-school approach to implementing the IMPP in their region. This decision was based on a set of three assumptions. Firstly, the belief that a beginning teacher cannot thrive in a dysfunctional school and that the principal/school leadership sets the "tone" for induction and mentoring practices for the school. This allows the whole school to embrace mentoring and create an environment where PRTs can learn and develop. Secondly, school management needs to give induction and mentoring status within the school. This affects how mentors and other teachers view mentoring which, in turn, gives rise to effective and efficient management of mentoring resources and release time. Thirdly, effective mentors are a necessary but not sufficient condition to enable sustainable shifts in induction and mentoring practices in a school or to achieve sustainable outcomes for PRTs. Sustainability and longer-term effects require induction and mentoring to be embedded within the school's vision, policies, practices and procedures. It must be a considered choice and commitment for the school.

Participants. Six schools were purposively selected, four from low and two from high socio-economic communities (two suburban intermediate, Years 7 and 8; two rural, Years 1 to 8; one integrated suburban, Years 1 to 8 ; one suburban, Years 1 to 6). A total of six principals, 17 mentors, 23 PRTs and up to 145 teaching staff were involved.

The professional development intervention. The intervention takes a systemic approach and the success of PRT induction is predicated on leaders taking an active interest in a PRT's knowledge, theoretical beliefs, and skill acquisition. In 2009, strategies to engage principals and leaders involved them and teaching staff: building on their knowledge of PRT development and induction; reviewing site-specific induction policy and programmes against a theoretical model of effective induction; goal setting; developing action plans; and working collaboratively to further school-specific induction and mentoring models and vision statements, drawing on the Council's draft 
guidelines. Knowledge building occurred through staff meetings and an educative mentoring course. At the end of the first year of the pilot, principals, mentors and school staff from the six schools met to share their learning experiences. Mentors participated in a 10-session programme on knowledge building, understanding and skill acquisition which focused on a conceptual understanding of educative mentoring. Participants carried out classroom observations and provided feedback and feed-forward through taped learning conversations with PRTs.

Research. The pilot uses a longitudinal mixed-methods approach, with questionnaires, interviews and taped conversations, to develop an understanding of effective induction and mentoring. It examines the role school leaders, mentors and teachers play in translating the national induction system (policy, resourcing, and guidelines) into school-based induction programmes; the understandings, attitudes and skills that educative mentors require to ensure that PRTs' learning focuses on valued student outcomes; how PRTs' learning and development is supported by leaders, mentors and school staff to meet the Registered Teacher Criteria; and the extent to which assessment practices affect confidence in the judgements made about the PRTs' ability to meet the Registered Teacher Criteria.

\section{The Secondary Education Pilot}

Nau te rourou, naku te rourou, ka ora ai te iwi

From your food basket, from my food basket, together we will strengthen the people

The Centre for Educational Development at Massey University is conducting the secondary education pilot, which aims to strengthen induction and mentoring in secondary schools in Taranaki-Wanganui, Manawatu-Rangitikei and Hawkes Bay. There are particular complexities in responsibility for supporting PRTs in secondary schools. These relate to multiple colleagues in supportive roles; with PRTs being supported by mentor teachers, PRT co-ordinators and specialist classroom teachers throughout their induction period. This proposal aimed to use these complexities to advantage by utilising the support networks within and across schools. The pilot has a particular emphasis on building mentoring capability through multiple partnerships, for example, schools are paired in each region to work 
together, new mentors are paired with experienced mentors, and the facilitators are paired across schools. The focus on multiple partnerships is one way Massey is ensuring sustainability for induction and mentoring practices in the region. Building on the previous experience of the Advisory Services in this area, the team was well placed to implement a model of support that would bring about changes in induction and mentoring as articulated in the Council's Draft Guidelines and the relevant literature.

Participants. Six rural and provincial secondary schools with rolls of between 330 and 770 students and deciles of between two and five are participating in the pilot. Schools were selected based on a history of employing PRTs and a limited capacity in mentoring. The facilitators work with a team of mentor teachers, PRT co-ordinators, specialist classroom teachers, and senior managers within and between pairs of schools. In some cases these roles overlap in individual people.

The professional development intervention. A key feature of this pilot is the focus on building mentoring capacity and capability. To achieve this, an action learning model of professional development has been implemented. This model is based on a needs analysis, selftracking matrix, and opportunities for discussion, reflection and feedback for next steps. It has used a mixture of workshops, facilitator visits, an online wiki and a mini-conference at the end of the first year that brought all participants together. The draft guidelines underpin the model and participants have provided critical feedback on them. In addition, those mentors participating in the first year of the pilot were paired with a new mentor in the second year, building capacity by increasing the number of trained mentor teachers in the schools. As with the other pilots, the Council's revised Registered Teacher Criteria have been used in the programme as the standards the PRTs are working towards to gain full registration, and therefore in shaping the mentoring of the PRTs. The participants have developed a selfassessment tool to chart a PRT's progress against the Registered Teacher Criteria. Participants have developed induction and mentoring plans in each school which have been shared across the participating schools, particularly between the partner schools. As part of these plans, schools have focused on strategies to train future mentors and help build sustainability. The wiki has enabled all participants to share progress and resources across the regions. 
Research. In addition to the evaluative data gathered as part of the overall IMPP, a team of researchers from the university is evaluating the effect of the pilot on policies and practices in each school.

\section{The Māori-Medium Education Pilot}

\section{Te Amorangi ki mua Te Hapai o ki muri}

The bearers of the prized treasure lead in front, the producers and supporter follow behind

The Māori-medium education pilot is conducted by Te Whare Wānanga o Awanuiāangi. In developing a professional development intervention for mentors and PRTs, Awanuiārangi first reviewed the Council's draft guidelines. The next step focused on developing a conceptual model appropriate for Māori medium settings, named Te Amorangi ki Mua, which is underpinned by the reciprocal concept of ako (the interaction between the teacher and the learner). Te pia (the PRT) is at the apex of the model, supported by te kaiako hapai (the mentor), who in turn is supported by te hapai o ki muri (network of agencies). All are supported by the concept and practice of ako, which is informed by a number of foundation principles such as manaakitanga (caring and sharing). Each of these principles has, in turn, been referenced to various combinations of the Registered Teacher Criteria.

Participants. The participants are teachers, professional leaders, mentors and PRTs from the Bay of Plenty region. A diverse range of institutions in early childhood education, primary and secondary schooling with a wide range of bilingual approaches to education are involved.

Professional development intervention. This pilot has evolved into a developmental project. In the first year, most of the professional development involved workshops focused on developing the model described above and the support material to be used by mentors (a mentoring handbook). That work led to the development of a selfreflective tool, based on the Registered Teacher Criteria. The next phase is development and implementation of a professional development programme for those providing the induction support to PRTs and evaluation of the effect of this programme.

Research. Research to date has included interviews in October with tumuaki and with a pou tautoko panel (advisory group) on the 
induction and mentoring support currently provided in their schools and early childhood education services, and further questions about mentoring put to participants at their workshops.

\section{The Evaluation of the Induction and Mentoring Pilot Project}

In addition to the research conducted by each contractor to inform and evaluate outcomes from their professional development programme, an independent national evaluation of the IMPP is in progress. Interim findings are discussed below. The evaluation is both formative and outcomes-based. Figure 1 (Teague \& Sankar, 2010, p. 14) provides an overview of the three phases of the external evaluation and common evaluative questions being used to monitor each pilot and make comparions across the pilots.

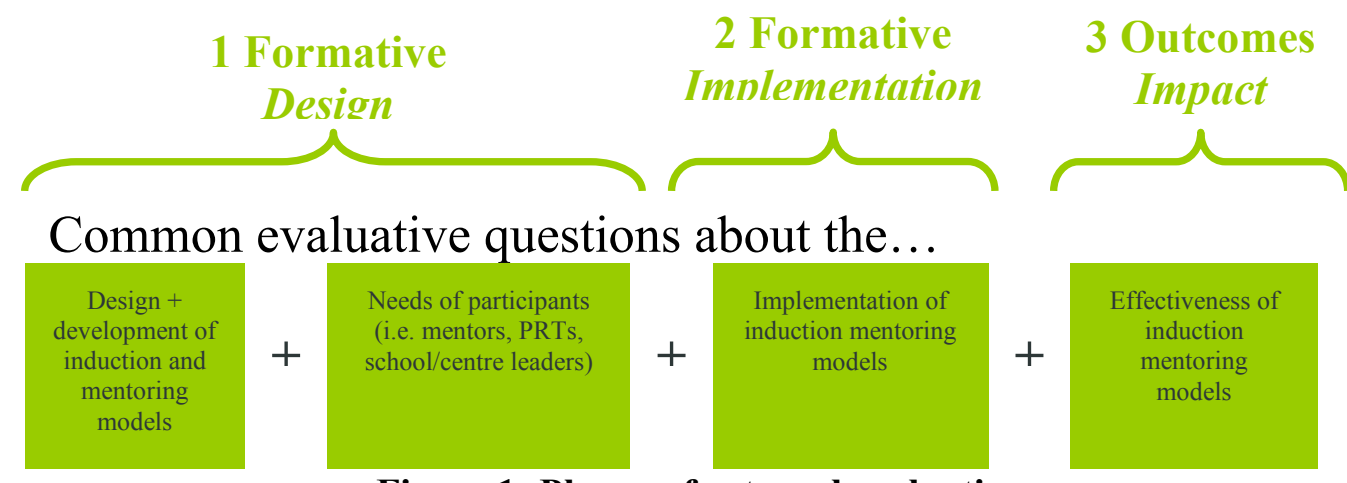

Figure 1: Phases of external evaluation

Because each pilot runs for a different length of time, the evaluation has been designed to be flexible to accommodate the different timeframes.

The data gathering has moved from general programme design to the more specific interactions with mentors and PRTs (see Figure 1). Interviews with pilot providers, school principals, mentors and PRTs are triangulated with findings from regular milestone reports submitted by providers. Initially this has examined the extent and ways the draft guidelines have influenced the development of each pilot's induction and mentoring model and the factors that may have enabled or constrained improvements in induction and mentoring practice (formative phase 1 and 2). This will build to a final evaluation of the extent and ways the draft guidelines and the approaches to induction and mentoring, trialled by the pilots, have contributed to improving mentor teacher practice and PRTs' induction experiences 
(outcomes phase 3). Ultimately this evaluation should identify factors in effective and sustainable induction and mentoring that the Council can use to enhance practices at a systems and policy level.

\section{Realising potential}

Despite finding some significant contextual differences within each sector which enable and inhibit practices, the evaluation of the IMPP identified some universal core skills, needs and outcomes sought from effective induction and mentoring practices. This confirms the relevance and utility of the draft guidelines for all pilots, although the feedback from Awanuiārangi indicates that Māori learning principles are missing from the draft guidelines. Awanuiārangi is preparing a handbook that may offer direction and insight in this regard. Nevertheless, the draft guidelines provide flexibility, and an overarching view of what constitutes effective induction and mentoring. Importantly they allow schools and early childhood education services to interpret the guidelines in their context and determine how best to implement them, given their particular issues and concerns.

Figure 2 (Teague \& Sankar, 2010, p. 55) illustrates the potential of the IMPP (the draft guidelines and the models of induction and mentoring support) to bring about desired shifts in induction and mentoring practices and to improve consistency and coherence in these practices across the sector. This diagram is at an aggregate level across the four pilots and shows the collective impact of the IMPP. 


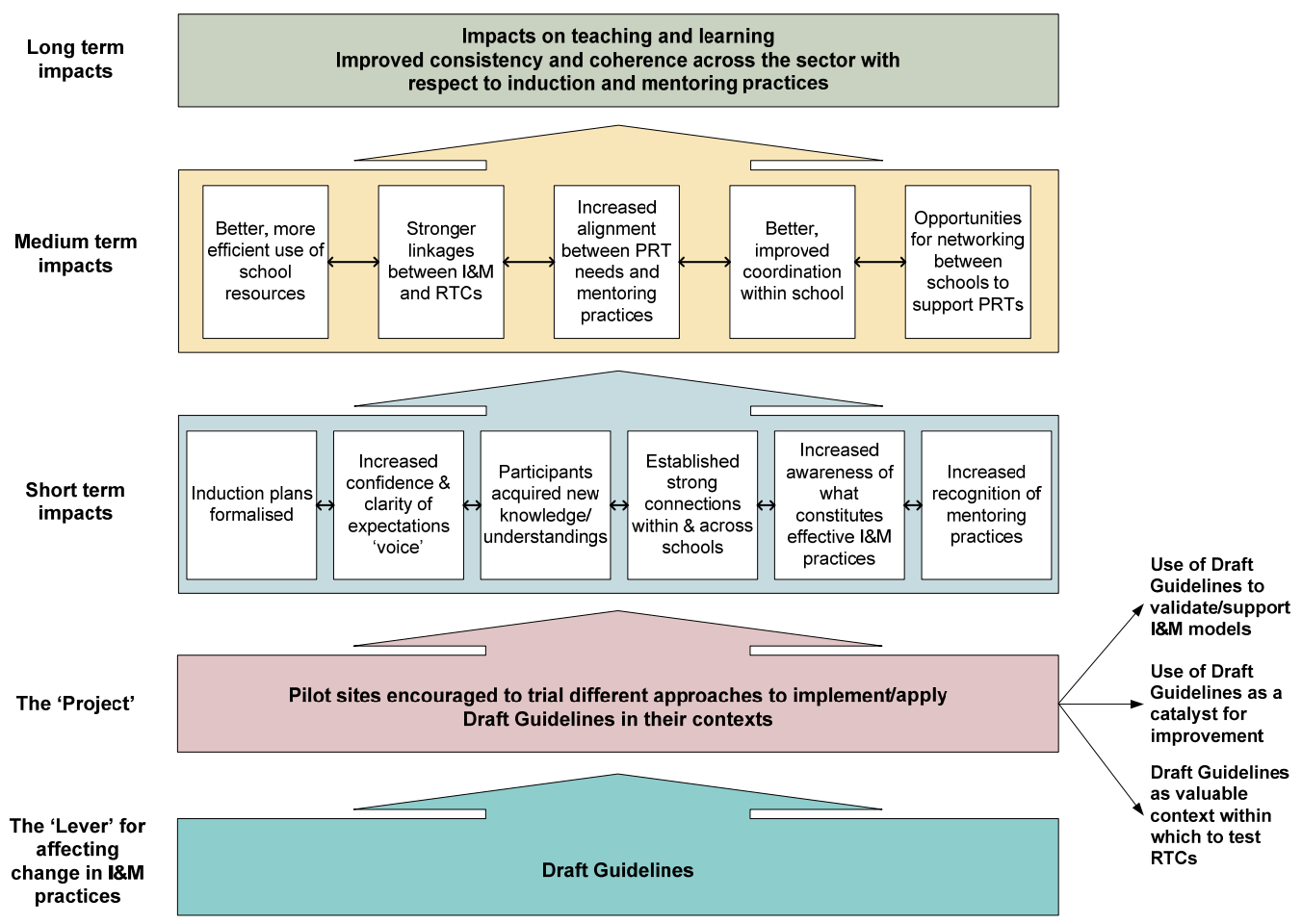

Figure 2: How IMPP works to bring about desired outcomes - an analytical model

The short-term impact or benefits of the IMMP have focused on individual and organisation changes. Schools and early childhood education services have formalised induction plans (with the exception of Māori medium, which is still under development) and built relationships across settings. At the individual level, professional leaders, mentor teachers, PRTs and other staff have new knowledge and understanding of what effective induction and mentoring is and confidence to fulfil their roles in the programme (see Figure 2). Further, these impacts have contributed to an increased recognition of induction and mentoring practices. Participating in the pilot put induction and mentoring practices in the spotlight, and services and schools paid more attention to them. The IMPP also influenced the connections between schools and services, and each of the four pilots attempted to tighten the connection between the workplace and mentors (in secondary through a paired partnership approach; in early childhood and Māori medium through regional workshops/hui; and in primary/intermediate through working with regional groups of schools). All participants have noted the value of being linked to other schools and services in order to share learning at a setting and individual level and to assist each other to reflect on practices. 
It is anticipated that these short-term impacts will contribute to achieving medium-term impacts for participants that lead to consistent, comprehensive induction practices. There should be more efficient use and co-ordination of resources, and frequent networking between settings. A clear alignment between the PRT and mentor teacher needs should be evident as well as links between the induction programme and the Registered Teacher Criteria (see Figure 2).

At this stage of the project, it is difficult to see if, in fact, the shortterm impacts will endure long enough for the medium-term outcomes to occur as anticipated. Notwithstanding, in the context of IMPP, the draft guidelines can be seen as a potential lever for effecting change in induction and mentoring practices. By encouraging and requiring pilots to trial the draft guidelines, the IMPP attempted to influence behaviours of both mentors and professional leaders to review their practice. The way in which the pilots used and implemented the draft guidelines in their contexts varied, with one using it to validate and support an induction and mentoring model, and others using it to develop or improve their model of induction and mentor teacher development. In all these situations, the draft guidelines acted as a catalyst for change. The pilots have shown how the potential value of PRT induction and mentoring, as set down in the guidelines, can be realised. Nevertheless, still to be addressed are the challenges and underlying paradox outlined below.

\section{Recognising paradox}

The evaluation has raised the issue of the sustainability of changes to practice (Teague \& Sankar, 2010). The pilots are resourced and have requirements to be met in a set timeframe. These factors predictably will have an effect on induction and mentoring programmes. Nevertheless, the IMPP has demonstrated that the draft guidelines can provide a clear path for change that contributes to improved consistency and coherence of induction and mentoring practices across the education sector. The IMPP analytical model (Figure 2) provides an insight into ways the draft guidelines may create a longerterm effect.

A key learning from the evaluation of the pilot is that the role of leaders in the facilitation of the on-site social processes surrounding the implementation of the draft guidelines is critical to enabling honest reflection by schools and services, mentors, and PRTs about current 
practices. The key role leaders play in sustaining policy change has been found in other studies (Sparks, 2005; Timperley, Wilson, Barrar \& Fung, 2008). Recognising the influence of the site-specific, collective interpretions of the guidelines is essential if the Council's aspirations of achieving a shift in induction and mentoring practices are to be realised.

While the draft guidelines clearly outline the desirable skills, knowledge and attributes for mentors, and indicate the areas in which mentors need to be trained and supported, early evaluation showed mentors found some skills more challenging than others. Combining assistance and assessment, for example, was seen as challenging. Although the pilots provided mentors with skills on how to have constructive learning conversations with PRTs, there was some anxiety evident. This was because of an underlying paradox; the tension between wanting to build a lasting relationship that is trusting, caring and professional and having to assess and measure PRTs' performance. Despite this, the draft guidelines, in combination with participation in the pilot, have acted as a catalyst for mentor selfassessment. Use and knowledge of the draft guidelines has helped mentors gain considerably more in-depth understanding, awareness and appreciation of what effective mentoring looks like and of the impact of their actions on PRTs. Before this, there was no formal mechanism which encouraged or facilitated mentor self-reflection and development.

\section{Conclusion}

The Council's IMPP has shown that national guidelines have the potential to influence schools and early childhood education organisations to promote high quality learning for both PRTs and mentors. There is still the issue of how to ensure leaders and teachers in all schools and early childhood education services take seriously the need to shift professional development from a culture of "fitting in" to a focus on teacher and children's learning, without jeopardising care and trust. What would it take for all leaders and mentors to combine support with an educative, transformative approach to mentor and PRT learning? Policy, guidelines and resourcing can make a difference; however, ultimately the onus is on leaders in each sector to respond to their unique situation. 
Despite the challenges, outlined above, committed leaders and mentors in schools and early childhood organisations do ensure PRTs are well supported and engaged in learning, within a community of learners. Even so, mentors are not necessarily trained or experienced in teaching adults how to teach. The pilots acknowledge that mentor teachers require knowledge of what it means to be an educative mentor and how to use this knowledge to assess PRTs against the Registered Teacher Criteria. Further, the pilots support Yusko and Feiman-Nemser's (2008) argument that mentors need to use a "full complement of mentoring tools". To be educative, mentors require sustained learning opportunities to use tools such as protocols for analysing students' and teachers' work, and engaging in learning conversations that provide meaningful feedback to effect student learning. In short, for PRTs to become effective teachers, they need to be led by teachers who are expert mentors.

When PRTs work in environments that neglect professional learning and focus primarily on support and advice, they will not reach their full potential as effective educators. The draft guidelines challenge practice but, if taken seriously, they do have the potential to create change to support teacher learning to meet the Registered Teacher Criteria and build career-long habits of professional reflection and learning. As the project and evaluation progresses, lessons learned from sector-specific contexts will provide further insight into changes that need to be made to policy and practice in support of a national educative and equitable approach to PRT induction and mentoring.

\section{Endnotes}

1. The Registered Teacher Criteria were finalised in October 2009. The pilots used the draft criteria in 2009 and the finalised criteria from 2010.

\section{Acknowledgements}

The project leaders for each pilot contract have informed and contributed to this article. We acknowledge these people in particular, and the people who work with them: Colleen Douglas, Massey University; Clare Wells, New Zealand Kindergartens Inc.; Kuni Jenkins, Te Whare Wānanga o Awanuiārangi; the team with Frances Langdon, the University of Auckland; and Meenakshi Sankar, Marinka Teague and Nicole Brown, Martin Jenkins Ltd.

The project is funded by the New Zealand Teachers Council, which established a steering group chaired by Dr Lexie Grudnoff. This group of professional and academic members monitor the progress of the pilots, provide feedback on all milestone reports, and comment on the key findings. In addition, a wider reference group from the education sector, and members of the Council itself, receive progress reports and provide feedback to the project. 


\section{The authors}

Frances Langdon is Head of School, Teaching Learning and Development at the University of Auckland. Her research focuses on teacher learning and development, particularly early career learning, induction, and mentoring. She is the principal investigator of the primary/intermediate induction and mentoring pilot.

Peter Lind is Director of the New Zealand Teachers Council and formerly Director of Teacher Education at Massey University.

Cynthia Shaw is Manager, Policy and Strategic Development at the New Zealand Teachers Council and formerly a teacher and senior manager involved in the secondary education sector.

Erin Pilcher is a Researcher working in the Policy and Strategic Development team at the New Zealand Teachers Council.

Contact Author: Frances Langdon, Faculty of Education, School of Teaching Learning and Development, University of Auckland, 74 Epsom Avenue, Auckland. Email: f.langdon@auckland.ac.nz. 


\section{References}

Aitken, H., Ferguson, P. B., Mcgrath, F., Piggot-Irvine, E., \& Ritchie, J. (2008). Learning to teach: Success case studies of teacher induction in Aotearoa New Zealand. Wellington: New Zealand Teachers Council.

Battersby, D. (1989). Beginning teacher scheme evaluation. Wagga Wagga, Australia: Riverina-Murray Institute of Higher Education.

Britton, E., Paine, L., Pimm, D., \& Raizen, S. (2003). Comprehensive teacher induction. Dordrecht: Kluwer Academic.

Cameron, M. (2006). Stage one: Literature review on teacher induction. Wellington: New Zealand Council for Educational Research.

Cameron, M. (2007). Learning to teach: A literature review of induction theory and practice. Wellington: New Zealand Teachers Council.

Cameron, M. (2009). Lessons from beginning teachers: Challenges for school leaders Wellington: NZCER Press.

Cameron, M., Dingle, R., \& Brooking, K. (2007). Learning to teach: A survey of provisionally registered teachers in Aotearoa New Zealand. Wellington: New Zealand Teachers Council.

Dewar, S., Kennedy, S., Staig, C., \& Lois, C. (2003). Recruitment and retention in New Zealand secondary schools. Wellington: Ministry of Education.

Feiman-Nemser, S. (2001). Helping novices learn to teach: Lessons from an exemplary support teacher. Journal of Teacher Education, 52, 17-30.

Gold, Y. (1996). Beginning teacher support: Attrition, mentoring and induction. In J. Sikula, T. Buttery \& E. Guyton (Eds.), Handbook of research on teacher education (Vol. 2, pp. 548-594). New York: Macmillan.

Mansell, R. (1996). Survey of year one and two teachers. Wellington: New Zealand Teacher Registration Board.

New Zealand Teachers Council. (2009a). Draft guidelines for induction and mentoring programmes and for mentor teacher development in Aotearoa New Zealand. Retrieved February 1, 2010, from http://www.teacherscouncil.govt.nz/policy/projects1.stm.

New Zealand Teachers Council. (2009b). Registered teacher criteria. Retrieved February 1, 2010, from http://www.teacherscouncil.govt.nz/policy/ projects1.stm.

Norman, P. J., \& Feiman-Nemser, S. (2005). Mind activity in teaching and mentoring. Teaching and Teacher Education, 21(6), 679-697.

Organisation for Economic Co-operation and Development. (2005). Teachers matter: Attracting, developing and retaining effective teachers. Paris: OECD. 
Renwick, M. (2001). Support for beginning teachers. Wellington: Ministry of Education.

Smith, T., \& Ingersoll, R. (2004). What are the effects of induction and mentoring on beginning teacher turnover? American Educational Research Journal, 41(3), 681-741.

Sparks, D. (2005). The gift that one generation of educators gives the next. In H. Portner (Ed.), Teacher mentoring and induction: The state of the art and beyond (pp. 241-244). Thousand Oaks: Corwin Press.

Strong, M. (2009). Effective teacher induction and mentoring: Assessing the evidence. New York: Teachers College Press.

Teague, M., \& Sankar, M. (2010). New Zealand Teachers Council Induction and Mentoring Pilot Project: Evaluation report (Commercial In Confidence). Wellington: Martin Jenkins.

Timperley, H., Wilson, A., Barrar, H., \& Fung, I. (2008). Teacher professional learning and development: Best evidence synthesis iteration (BES). Wellington: Ministry of Education.

Wong, H. K. (2005). New teacher induction: The foundation for comprehensive, coherent, and sustained professional development In H. Portner (Ed.), Teacher mentoring and induction (pp. 41-58). Thousand Oaks: Corwin Press.

Yusko, B., \& Feiman-Nemser, S. (2008). Embracing contraries: Combining assistance and assessment in new teacher induction. The Teachers College Record, 110(5), 923-953. 